\title{
Cosmological solutions in multiscalar field theory
}

\author{
N. Dimakis ${ }^{1, \mathrm{a}}{ }^{\mathbb{D}}$, A. Paliathanasis $^{2, \mathrm{~b}}$, Petros A. Terzis ${ }^{3, \mathrm{c}}$, T. Christodoulakis ${ }^{3, \mathrm{~d}}$ \\ ${ }^{1}$ Center for Theoretical Physics, College of Physical Science and Technology, Sichuan University, Chengdu 610065, China \\ ${ }^{2}$ Institute of Systems Science, Durban University of Technology, POB 1334, Durban 4000, South Africa \\ ${ }^{3}$ Nuclear and Particle Physics Section, Physics Department, University of Athens, 15771 Athens, Greece
}

Received: 21 April 2019 / Accepted: 9 July 2019 / Published online: 20 July 2019

(C) The Author(s) 2019

\begin{abstract}
We consider a cosmological model with two scalar fields minimally coupled to gravity which have a mixed kinetic term. Hence, Chiral cosmology is included in our analysis. The coupling function and the potential function, which depend on one of the fields, characterize the model we study. We prove the existence of exact solutions that are of special interest for the cosmological evolution. Furthermore, we provide with a methodology that relates the scale factor behaviour to the free functions characterizing the scalar field kinetic term coupling and potential. We derive the necessary conditions that connect these two functions so that the relative cosmological solutions can be admitted. We find that unified dark matter and dark energy solutions are allowed by the theory in various scenarios involving the aforementioned functions.
\end{abstract}

\section{Introduction}

From the detailed analysis of the recent cosmological data $[1-3]$ it has been made clear the necessity for the introduction of an exotic fluid term with negative pressure in Einstein's General Relativity. The role of this exotic matter source is to explain the acceleration phases of the universe. So far its nature is unknown and it has only been observed indirectly; it is thus referred in the literature as dark energy. The contribution of the dark energy in the observable universe is approximately $\sim 70 \%$ while the rest $\sim 30 \%$ corresponds mostly to dark matter (with a smaller contribution by baryonic matter and radiation). Dark matter is a pressureless fluid source (dust) that also has not been observed directly and which was introduced in order to explain the observed rotation curves of the galaxies.

\footnotetext{
a e-mails: nsdimakis@scu.edu.cn; nsdimakis@gmail.com

be-mail: anpaliat@phys.uoa.gr

c e-mail: pterzis@phys.uoa.gr

de-mail: tchris@phys.uoa.gr
}

Although the cosmological constant is the most simple dark energy candidate, $\Lambda$-cosmology suffers by two major drawbacks: (a) fine tuning and (b) the coincidence problem [4,5]. A quite wide-known alternative approach on the cosmological constant is scalar field cosmology [6]. Scalar fields can be introduced either minimally or nonminimally coupled to gravity. In what regards the minimally coupled case, the general solution for an arbitrary potential function has been obtained for all types of Friedmann-Lemaître-RobertsonWalker (FLRW) spacetimes: open, closed and spatially flat [7]. Scalar fields can also be used to incorporate - in an equivalent picture - the new degrees of freedom provided by the higher-order derivatives in extended theories of gravity [813]. Apart from the application of scalar fields in order to account for the late-time acceleration of the universe, they have been additionally used as unified dark energy models [14-24].

With the term scalar field cosmology usually someone refers to quintessence theories. However, there are many alternatives like, phantom fields, Hordenski gravity [10], or even multi-scalar field models. The latter have a broad range of applications as they can provide alternative models of inflation, namely: hybrid inflation, $\alpha$-attractors, double inflation and many others [25-36]. Quintom scalar field models consist of two scalar fields, a quintessence field and a phantom field, for a review we refer the reader in [37]. The main characteristic of the quintom models is that in general the two scalar fields are minimally coupled and only a few models have been proposed where an interaction term between the two fields is introduced in the potential [37]. While a quintom model with a mixed-kinetic term has been introduced before [38], due to the fact that the geometry of the space where the kinetic terms of the scalar field form is flat there can always be defined new scalar fields so as to neglect the mixed kinetic term.

Multi-scalar field models where interaction exists in the kinetic term of the Lagrangian is where this work focuses. A 
specific family of these models, where the two scalar fields defined into a two-dimensional space of constant nonvanishing curvature, are the Chiral cosmological models $[33,39,40]$ which are directly related with the non-linear sigma cosmological model [41-43]. In this work we consider two-scalar field cosmological model where the one field is quintessence and the second field interact with the quintessence only in the kinetic term. The model consists of two unknown functions, the potential which drives the dynamics of the quintessence and the interaction between the fields. For this cosmological model we prove the existence of particular solutions of great interest and determine the conditions that they impose in the functions characterizing the theory. Among the solutions we study are: scaling solutions, de Sitter solutions and that of asymptotic de Sitter spacetimes with or without cosmological singularity. The last cosmological scenario is very interesting since $\Lambda \mathrm{CDM}$ cosmology is recovered. What is more, the determination of exact or analytical solutions is important because the main properties of a given model can be determined without suffering from the problem of selecting the initial conditions. Moreover, when we use numerical methods to solve a given dynamical system, we know that the numerical simulations describe real trajectories of the problem. Some results with exact and analytic solutions in multi scalar field cosmology are given in [39,40,44-57].

In this work we study a two scalar field cosmological system. We are mainly interested in presenting a method for providing exact solutions together with the class of theories to which they correspond. This is realized by deciding on a specific scale factor behaviour and then providing algorithmically the relation between the theory's free functions that permit such a solution. Thus, we are able to derive - within the type of model we study - which specific relation between the kinetic coupling and the scalar field potential allows for certain interesting cosmological solutions.

The outline of the paper is as follows: In Sect. 2 we briefly present the cosmological model that we consider and derive the gravitational field equations in the case of a spatially flat FLRW spacetime. In Sect. 3 we prove the existence, under conditions, of four important families of scale factor solution of cosmological interest. More specifically, we determine solutions for scaling solutions, de Sitter solution, asymptotically de Sitter spacetimes and exponentials of arbitrary powers of time. There exists a case where the scalar field potential and the interaction function are exponential with different exponents, where the scalar factor is given by a power-law function in which the acceleration of the universe can be described, including the inflationary era. For that specific cosmological model in Sect. 4 we perform a detailed analysis on the dynamics of field equations by studying the existence and the stability of critical points. Finally, in Sect. 5 we discuss our results and draw our conclusions.

\section{Field equations}

Let us consider the action integral

$$
\begin{aligned}
S= & \int d^{4} x \sqrt{-g} \mathcal{L}\left(R\left(x^{\mu}\right), \phi\left(x^{\mu}\right), \psi\left(x^{\mu}\right),\right. \\
& \left.\times \nabla_{\nu} \phi\left(x^{\mu}\right), \nabla_{\nu} \psi\left(x^{\mu}\right)\right)
\end{aligned}
$$

characterized by the Lagrangian density

$\mathcal{L}=\frac{1}{2} R-\frac{1}{2} \nabla_{\mu} \phi \nabla^{\mu} \phi-\frac{F(\phi)}{2} \nabla_{\mu} \psi \nabla^{\mu} \psi-V(\phi)$,

where we have adopted the geometric units $8 \pi G=c=1$. As usual, $g$ is used to symbolize the determinant of the spacetime metric, while $R$ is reserved for the scalar curvature.

From (2.2) it is obvious that we consider two scalar fields $\phi\left(x^{\mu}\right)$ and $\psi\left(x^{\mu}\right)$ as the matter content of the theory. At the same time we assume an interacting function $F\left(\phi\left(x^{k}\right)\right)$ and a potential $V\left(\phi\left(x^{\mu}\right)\right)$ that depend solely on the scalar field $\phi\left(x^{\mu}\right)$.

The field equations produced in this setting, by variation with respect to the metric $g_{\mu \nu}\left(x^{\mu}\right)$ and the scalars $\phi\left(x^{\mu}\right)$, $\psi\left(x^{\mu}\right)$ are respectively:

$$
\begin{aligned}
R_{\mu \nu}-\frac{1}{2} g_{\mu \nu} R & =T_{\mu \nu} \\
\nabla_{\mu} \nabla^{\mu} \phi-V^{\prime}(\phi)-\frac{1}{2} F^{\prime}(\phi) \nabla_{\mu} \psi \nabla^{\mu} \psi & =0 \\
\nabla_{\mu}\left(F(\phi) \nabla^{\mu} \psi\right) & =0,
\end{aligned}
$$

where $R_{\mu \nu}$ is the Ricci tensor and the prime denotes a derivative with respect to $\phi$. The energy momentum tensor that appears in (2.3a) is given by

$$
\begin{aligned}
T_{\mu \nu}= & \nabla_{\mu} \phi \nabla_{\nu} \phi-\frac{1}{2}\left(\nabla^{\kappa} \phi \nabla_{\kappa} \phi\right) g_{\mu \nu}-V(\phi) \\
& +F(\phi) \nabla_{\mu} \psi \nabla_{\nu} \psi-\frac{F(\phi)}{2}\left(\nabla^{\kappa} \psi \nabla_{\kappa} \psi\right) g_{\mu \nu} .
\end{aligned}
$$

Due to the fact that we choose the functions $F\left(\phi\left(x^{\mu}\right)\right)$, $V\left(\phi\left(x^{\mu}\right)\right)$ to depend only on $\phi\left(x^{\mu}\right)$, the action (2.1) is bound to remain form invariant under the global transformation $\psi\left(x^{\mu}\right) \mapsto \psi\left(x^{\mu}\right)+\varepsilon$, in which $\varepsilon$ is a constant. The corresponding conservation law is already obvious from the equation of motion (2.3) which implies

$\partial_{\mu}\left[\sqrt{-g} F(\phi) \nabla^{\mu} \psi\right]=0$,

where the term inside the bracket is the Noether current corresponding to the aforementioned symmetry transformation.

We consider a spatially flat FLRW spacetime with line element

$$
\begin{aligned}
d s^{2}= & g_{\mu \nu} d x^{\mu} d x^{\nu}=-N^{2}(t) d t^{2}+a(t)^{2} \\
& \times\left(d r^{2}+r^{2} d \theta^{2}+r^{2} \sin ^{2} \theta d \varphi^{2}\right),
\end{aligned}
$$


where $a(t)$ is the scale factor of the universe and $N(t)$ is the lapse function. For the scalar fields we assume that they inherit the symmetries of the spacetime which means $\phi=$ $\phi(t)$ and $\psi=\psi(t)$. Under this assumption the gravitational plus matter field equations reduce to the following set of ordinary differential equations

$$
\begin{aligned}
& \frac{1}{2 N^{2}}\left[F(\phi) \dot{\psi}^{2}+\dot{\phi}^{2}-\frac{6 \dot{a}^{2}}{a^{2}}\right]+V(\phi)=0 \\
& \frac{1}{2 N^{2}}\left[-\frac{4 \ddot{a}}{a}+\frac{4 \dot{a} \dot{N}}{a N}-\frac{2 \dot{a}^{2}}{a^{2}}-F(\phi) \dot{\psi}^{2}-\dot{\phi}^{2}\right]+V(\phi)=0
\end{aligned}
$$

$\frac{1}{2 N^{2}}\left[2 \ddot{\phi}+\frac{6 \dot{a} \dot{\phi}}{a}-F^{\prime}(\phi) \dot{\psi}^{2}-\frac{2 \dot{N} \dot{\phi}}{N}\right]+V^{\prime}(\phi)=0$

$\frac{d}{d t}\left(\frac{a^{3} F(\phi) \dot{\psi}}{N}\right)=0$,

where the dot signifies derivative with respect to the parameter $t$.

From the last equation the corresponding conserved quantity of the reduced system becomes apparent since it implies

$Q:=\frac{a^{3} F(\phi) \dot{\psi}}{N}=c_{1}$,

with $c_{1}$ being a constant of integration.

It is interesting to note that the same situation can be reproduced in the context of a minisuperspace approximation through the Lagrangian function

$L=-\frac{1}{2 N}\left(6 a \dot{a}^{2}-a^{3} \dot{\phi}^{2}-a^{3} F(\phi) \dot{\psi}^{2}\right)-N a^{3} V(\phi)$,

whose Euler-Lagrange equations are equivalent to (2.7) and of course its symmetries lead to the same conserved charge $Q=\frac{\partial L}{\partial \dot{\psi}}$.

We now proceed with our analysis by investigating the conditions under which certain interesting gravitational solutions may emerge. In what follows we adopt the gauge choice $N=1$ so that the results are expressed directly in a cosmological time.

\section{Exact solutions}

The gravitational field equations form a constraint system of nonlinear second-order differential equations for the three dependent variables $\{a(t), \phi(t), \psi(t)\}$. There are many alternative ways to study the dynamics and the evolution of the field equations. In this work we are interested in the existence of exact solutions. More specifically, we assume that the physical space is specified by particular functional forms for the scalar factor $a(t)$ which describe various eras in the evolution of the universe. Such an analysis is important because it provides information for the existence of various cosmological eras for the specific cosmological model. In addition to this, the cosmological validity of each model can be studied directly from such an analysis.

From the conserved quantity (2.8) we easily deduce that

$\dot{\psi}=\frac{c_{1}}{a^{3} F(\phi)}$.

By adding (2.7a) and (2.7b) the relation

$V(\phi)=\frac{\ddot{a}}{a}+\frac{2 \dot{a}^{2}}{a^{2}}$

is obtained (recall that we have set $N=1$ ). At this point we have acquired the potential $V(\phi)$ as a pure function of $t$. We continue our analysis by considering several interesting situations regarding the scale factor.

\subsection{Power law solutions}

In this case we are interesting in studying the conditions that a solution of the form

$a(t)=t^{\sigma}, \quad \sigma \in \mathbb{R}$,

can set over the theory. The power-law solution (3.3) is important, because it can describe the matter dominated era solution $\left(\sigma=\frac{2}{3}\right)$, the radiation era solution $\left(\sigma=\frac{1}{2}\right)$, or the early accelerated phase of the universe when $\sigma>1$.

For this choice of scale factor the space-time exhibits a curvature singularity at $t=0$ for any value of $\sigma$. With the help of (3.3) relation (3.2) results in

$V(\phi)=\frac{\sigma(3 \sigma-1)}{t^{2}}$.

This leads us to consider two distinct situations depending on the value of $\sigma$.

\subsubsection{Case $\sigma \neq \frac{1}{3}$}

We start by considering that the potential $V(\phi)$ cannot be zero, which according to (3.4) corresponds to $\sigma \neq \frac{1}{3}$. By inverting Eq. (3.4) we obtain $t$ as a function of $\phi$, i.e.

$t= \pm \frac{\sqrt{\sigma} \sqrt{3 \sigma-1}}{\sqrt{V(\phi)}}$. 
If we additionally take the derivative of (3.4) with respect to time, the relation

$V^{\prime}(\phi) \dot{\phi}=\frac{2(1-3 \sigma) \sigma}{t^{3}} \Rightarrow \dot{\phi}=\frac{2 \sigma(1-3 \sigma)}{t^{3} V^{\prime}(\phi)}$,

emerges. Direct substitution of (3.1), (3.5) and (3.6) into the constraint Eq. (2.7a) leads to the expression

$$
F(\phi)=c_{1}^{2} \sigma^{1-3 \sigma}(3 \sigma-1)^{1-3 \sigma} \frac{V(\phi)^{3 \sigma-1} V^{\prime}(\phi)^{2}}{2\left(\sigma V^{\prime}(\phi)^{2}-2 V(\phi)^{2}\right)},
$$

which involves only the two functions of $\phi$. This is how $F(\phi)$ and $V(\phi)$ have to be related for a power law solution of the form (3.3) with $\sigma \neq \frac{1}{3}$ to be admitted by the system. Any choice for the potential $V(\phi)$ leads to the corresponding expression of the coupling $F(\phi)$ needed for such a solution. Let us proceed with the study of two particular examples.

a. Example 1: Power law potential.

Let us set $V(\phi)=V_{0} \phi^{\kappa}$, with $V_{0}$ and $\kappa$ constants. Then, (3.7) implies

$F(\phi)=F_{0} \frac{\phi^{\kappa(3 \sigma-1)}}{2\left(\kappa^{2} \sigma-2 \phi^{2}\right)}$,

where with $F_{0}$ we re-parametrized the constant of integration $c_{1}$ corresponding to the conserved charge $Q$. The two constants are related through the expression

$c_{1}=\sqrt{F_{0}} \kappa^{-1} \sigma^{\frac{1}{2}(3 \sigma-1)}(3 \sigma-1)^{\frac{1}{2}(3 \sigma-1)} V_{0}^{\frac{1}{2}(1-3 \sigma)}$.

By setting (3.1), (3.3), (3.8) and $V(\phi)=V_{0} \phi^{\kappa}$ into the constraint Eq. (2.7a) we can solve the latter with respect to $\dot{\phi}$. In particular, we obtain:

$$
\begin{aligned}
\dot{\phi}= & \pm\left[\frac{2 \sigma^{3 \sigma-1}(3 \sigma-1)^{3 \sigma-1} V_{0}^{1-3 \sigma}}{\kappa^{2}} t^{-6 \sigma} \phi^{\kappa(1-3 \sigma)}\right. \\
& \left.\left(2 \phi^{2}-\kappa^{2} \sigma\right)+\frac{6 \sigma^{2}}{t^{2}}-2 V_{0} \phi^{\kappa}\right]^{\frac{1}{2}}
\end{aligned}
$$

Substitution of the $\dot{\phi}$ we derived and of all the rest of the previous mentioned expressions into Eq. (2.7b) leads to an algebraic solution for the scalar field $\phi$ :

$\phi(t)=\left(\frac{\sigma(3 \sigma-1)}{V_{0}}\right)^{\frac{1}{\kappa}} t^{-\frac{2}{\kappa}}$.
Consequently, the scalar field $\psi$ can be easily obtained now from (3.1) with a simple integration. The solution reads:

$$
\begin{aligned}
\psi(t) & =\psi_{0}+2 \sigma^{-\frac{3 \sigma}{2}}(3 \sigma-1)^{\frac{1}{2}-\frac{3 \sigma}{2}} V_{0}^{-\frac{-3 \kappa \sigma+\kappa+4}{2 \kappa}} t^{3 \sigma-1} \\
& \times\left(\frac{2(3 \sigma-1)^{2 / \kappa} \sigma^{\frac{2}{\kappa}+\frac{1}{2}} t^{-4 / \kappa}}{\kappa(1-3 \sigma)+4}+\frac{\kappa \sigma^{3 / 2} V_{0}^{2 / \kappa}}{3 \sigma-1}\right) F_{0}^{-\frac{1}{2}},
\end{aligned}
$$

where $\psi_{0}$ is a constant of integration. One can easily verify that the $\phi(t)$ and $\psi(t)$ as given by (3.11) and (3.12), together with the scale factor (3.3) satisfy the field Eq. (2.7) for the power law potential under consideration and the coupling function (3.8). We have thus obtained the solution for the theory, which admits a power law solution for the scale factor and for a power law potential $V$ with respect to $\phi$.

\section{b. Example 2: Exponential potential.}

We repeat the same procedure only this time we use a potential of the form $V(\phi)=V_{0} e^{\lambda \phi}$ with $\lambda$ a constant. The compatible (for a power law $a(t)$ ) coupling function $F(\phi)$ now becomes

$F(\phi)=F_{0} e^{\lambda(3 \sigma-1) \phi}$,

where $F_{0}$ is connected to the $c_{1}$ that we saw previously through

$c_{1}=\sqrt{2} \sqrt{F_{0}} \lambda^{-1} \sigma^{\frac{3 \sigma}{2}-\frac{1}{2}}(3 \sigma-1)^{\frac{3 \sigma}{2}-\frac{1}{2} \sqrt{\lambda^{2} \sigma-2}} V_{0}^{\frac{1}{2}-\frac{3 \sigma}{2}}$.

We notice that unlike the power law potential case, here, an exponential potential needs also an exponential coupling function to produce a scale factor of the form (3.3). As previously done, we substitute (3.1), (3.3), (3.13) and $V(\phi)=V_{0} e^{\lambda \phi}$ into the constraint Eq. (2.7a). From the latter we obtain the expression for $\dot{\phi}$. Subsequently, we substitute the latter into Eq. (2.7b) and we get an algebraic solution for $\phi(t)$ which this time reads

$\phi(t)=\frac{1}{\lambda} \ln \left(\frac{\sigma(3 \sigma-1)}{t^{2} V_{0}}\right)$.

The direct integration of (3.1) leads to

$$
\begin{aligned}
\psi(t)= & \psi_{0} \\
& +\frac{\sigma^{\frac{1}{2}-\frac{3 \sigma}{2}}(3 \sigma-1)^{-\frac{3 \sigma}{2}-\frac{1}{2}} \sqrt{2 \lambda^{2} \sigma-4} V_{0}^{\frac{3 \sigma}{2}-\frac{1}{2}}}{\sqrt{F_{0}} \lambda} t^{3 \sigma-1} .
\end{aligned}
$$

Again, it is easy to check that the above expressions satisfy the field equations for the choice of potential and the corresponding coupling function (3.13). After these two examples 
we can proceed with the case that we excluded from the power law solution of the scale factor.

\subsubsection{Case $\sigma=\frac{1}{3}$}

This means that $a(t)=t^{\frac{1}{3}}$, which corresponds to a potential $V(\phi)=0$. The function $F(\phi)$ remains arbitrary with the solution being described by the equations

$\dot{\psi}=\frac{c_{1}}{t F(\phi)}$

$\dot{\phi}= \pm \frac{1}{t}\left(\frac{2}{3}-\frac{c_{1}^{2}}{F(\phi)}\right)^{\frac{1}{2}}$

The latter set implies that the following relation exists between the two scalar fields

$\psi(\phi)=\psi_{0} \pm \int \frac{\sqrt{3} c_{1}}{\sqrt{F(\phi)} \sqrt{2 F(\phi)-3 c_{1}^{2}}} d \phi$

with $\psi_{0}$ being a constant. The function $F(\phi)$ can be chosen arbitrary and the solution can be obtained through a simple integration since (3.17) yields

$t=\exp \left( \pm \int\left(\frac{2}{3}-\frac{c_{1}^{2}}{F(\phi)}\right)^{-\frac{1}{2}} d \phi\right)$

\subsection{De Sitter universe}

In this case we introduce a scale factor of the form $a(t)=e^{\lambda t}$, where $\lambda$ is a constant. Later in our analysis we shall treat the more general case $a(t)=e^{\lambda t^{\mu}}$, but here we want to distinguish the particular solution corresponding to $\mu=1$ due to its importance in cosmology. The choice $a(t)=e^{\lambda t}$ for the scale factor leads (3.2) to become

$V(\phi)=3 \lambda^{2}$

Hence, the potential plays the role of a pure cosmological constant.

The constraint equation with the use of (3.1), which now reads

$\dot{\psi}=\frac{c_{1} e^{-3 \lambda t}}{F(\phi)}$

yields

$\dot{\phi}= \pm \frac{c_{1} e^{-3 \lambda t}}{\sqrt{-F(\phi)}}$ where $F(\phi)$ can be any arbitrary function of $\phi$. As in the special case of the previous section, we can deduce a relation between $\psi$ and $\phi$ since

$\frac{\dot{\psi}}{\dot{\phi}}= \pm \frac{1}{\sqrt{-F(\phi)}} \Rightarrow \psi=\psi_{0} \pm \int \frac{1}{\sqrt{-F(\phi)}} d \phi$

Equation (3.23) can serve to obtain $t$ as a function of $\phi$ and it results in

$t=\frac{1}{3 \lambda} \ln \left(\frac{\mp c_{1}}{3 \lambda \int \sqrt{-F(\phi)} d \phi}\right)$.

We notice that the previous expressions for $\dot{\psi}$ and $\dot{\phi}$ from (3.23) and (3.22) are such so that the sum of the kinetic terms corresponding to the matter content of the Lagrangian (2.9) is zero, i.e.

$\frac{a^{3} F(\phi) \dot{\psi}^{2}}{2 N}+\frac{a^{3} \dot{\phi}^{2}}{2 N}=\frac{1}{2} e^{3 \lambda t}\left(F(\phi) \dot{\psi}^{2}+\dot{\phi}^{2}\right)=0$.

Thus, it is necessary for one of the kinetic terms to change its sign. This was expected from the moment that we noticed that the choice $a(t)=e^{\lambda t}$ led to a potential that is a constant. We already know that such a space-time is a pure cosmological constant solution, hence any other contributions should in principle cancel each other in the Lagrangian. The only other possibility in the theory under consideration would the trivial solution of $\phi$ and $\psi$ being constants.

In the particular case where $F(\phi)=$ const. We have the typical quintessense - phantom pair that is usually encountered in quintom scenarios. In their generality quintom cosmological models are constructed so that the boundary $w=$ -1 is crossed [58,59], where $w$ is the usual equation of state parameter. In this paradigm we are trivially on this boundary with the kinetic energies being cancelling each other for every $t$.

\subsection{Asymptotically de Sitter space-time}

In this section we examine a couple of cases where the spacetime can be considered as being asymptotically de Sitter in the sense that, locally, the metric becomes that of a positive cosmological constant solution in the limit $t \rightarrow \pm \infty$.

\subsubsection{Space-time without singularity}

We adopt for the scale factor the choice $a(t)=(\cosh (t))^{\alpha}$. The resulting spacetime has its curvature scalars regular both at $t \rightarrow 0$ and $t \rightarrow \pm \infty$. We can distinguish two cases depending on whether the constant $\alpha$ takes the value $\frac{1}{3}$ or not. We start from the generic case, so we assume $a \neq \frac{1}{3}$. 
Under this assumption (3.2) becomes

$V(\phi)=\alpha+\alpha(3 \alpha-1) \tanh ^{2}(t)$,

which can be inverted to

$t= \pm \operatorname{arctanh}\left(\frac{\sqrt{V(\phi)-\alpha}}{\sqrt{\alpha(3 \alpha-1)}}\right)$.

For large values of $t$, the potential (3.27) becomes $V(\phi) \simeq$ $3 \alpha^{2}$ from where the de Sitter solution $a(t) \simeq e^{\alpha t}$ is recovered.

If we take the total derivative with respect to time, equation (3.27) leads to

$\dot{\phi}=\frac{2 \alpha(3 \alpha-1) \tanh (t)}{\cosh (t)^{2} V^{\prime}(\phi)}$.

Substitution of (3.28), (3.29) and (3.1) into the constraint Eq. (2.7a) provides us with a relation between the two functions of $\phi$

$F(\phi)=\frac{c_{1}^{2} \alpha^{1-3 \alpha}(1-3 \alpha)^{1-3 \alpha}\left(V(\phi)-3 \alpha^{2}\right)^{3 \alpha-1} V^{\prime}(\phi)^{2}}{2\left(6 \alpha^{3}-\alpha V^{\prime}(\phi)^{2}-2(3 \alpha+1) \alpha V(\phi)+2 V(\phi)^{2}\right)}$.

For completeness, we need to also consider the case $\alpha=\frac{1}{3}$. When this value for the power of $\cosh (t)$ is adopted, relation (3.2) results in a constant potential $V(\phi)=\frac{1}{3}$. The constraint Eq. (2.7a) and the first integral $Q$ yield:

$\dot{\psi}=\frac{c_{1}}{F(\phi) \cosh (t)}$

$\dot{\phi}= \pm \frac{1}{\cosh (t)} \sqrt{-\frac{c_{1}^{2}}{F(\phi)}-\frac{2}{3}}$.

From the above we extract easily the relation

$$
\begin{aligned}
\dot{\psi} \dot{\phi} & = \pm \frac{c_{1}}{\sqrt{-\frac{1}{3} F(\phi)\left(3 c_{1}^{2}+2 F(\phi)\right)}} \Rightarrow \psi \\
& =\psi_{0} \pm \int \frac{c_{1}}{\sqrt{-\frac{1}{3} F(\phi)\left(3 c_{1}^{2}+2 F(\phi)\right)}} d \phi .
\end{aligned}
$$

What is more, Eq. (3.31) is separable and we can easily derive $t$ in terms of $\phi$ for any (admissible) function $F(\phi)$,

$t=2 \tanh ^{-1}\left[\tan \left( \pm \frac{1}{2} \int \frac{1}{\sqrt{-\frac{c_{1}^{2}}{F(\phi)}-\frac{2}{3}}} d \phi\right)\right]$.

\subsubsection{Space-time with a curvature singularity}

If we consider a scale factor of the form $a(t)=(\sinh (t))^{\alpha}$ we obtain a space-time that possesses a curvature singularity at $t \rightarrow 0$, as is evident by the Ricci scalar that reads

$R=\frac{6 \alpha(\alpha+\alpha \cosh (2 t)-1)}{\sinh ^{2}(t)}$.

Once more we have to distinguish cases depending on the parameter $\alpha$. We start by considering $\alpha \neq \frac{1}{3}$. The same analysis that we followed previously results in the potential

$V(\phi)=\alpha+\alpha(3 \alpha-1) \operatorname{coth}^{2}(t)$,

which in its turn leads to

$t= \pm \operatorname{arccoth}\left(\frac{\sqrt{V(\phi)-\alpha}}{\sqrt{\alpha(3 \alpha-1)}}\right)$.

and

$\dot{\phi}=\frac{2 \alpha(1-3 \alpha) \operatorname{coth}(t)}{\sinh (t)^{2} V^{\prime}(\phi)}$.

With the help of the above the constraint Eq. (2.7a) gives rise to

$F(\phi)=-\frac{c_{1}^{2} \alpha^{1-3 \alpha}(3 \alpha-1)^{1-3 \alpha}\left(V(\phi)-3 \alpha^{2}\right)^{3 \alpha-1} V^{\prime}(\phi)^{2}}{2\left(6 \alpha^{3}-\alpha V^{\prime}(\phi)^{2}-2(3 \alpha+1) \alpha V(\phi)+2 V(\phi)^{2}\right)}$,

which for integer values of $\alpha$ can be either equal to (3.30) or opposite of it. In particular, if $\alpha$ is an odd number (3.39) is the opposite of (3.30). On the other hand, if it is even, then the two expressions yield the same result. An interesting value for the parameter $\alpha$ is that of $\alpha=\frac{2}{3}$, where the model mimics the $\Lambda$-cosmology.

Finally, we conclude this section by considering the $\alpha=\frac{1}{3}$ case. Once more the potential assumes the constant value $V(\phi)=\frac{1}{3}$, while for the two scalar fields we have

$$
\begin{aligned}
\dot{\psi} & =\frac{c_{1}}{F(\phi) \sinh (t)} \\
\dot{\phi} & = \pm \frac{1}{\sinh (t)} \sqrt{\frac{2}{3}-\frac{c_{1}^{2}}{F(\phi)}} .
\end{aligned}
$$

By combining the last two relations we get

$$
\begin{aligned}
\frac{\dot{\psi}}{\dot{\phi}} & = \pm \sqrt{\frac{3 c_{1}^{2}}{F(\phi)\left(2 F(\phi)-3 c_{1}^{2}\right)}} \Rightarrow \psi \\
& =\psi_{0} \pm \int \sqrt{\frac{3 c_{1}^{2}}{F(\phi)\left(2 F(\phi)-3 c_{1}^{2}\right)}} d \phi,
\end{aligned}
$$


while (3.41) implies

$t=2 \tanh ^{-1}\left[\exp \left( \pm \int \frac{1}{\sqrt{\frac{2}{3}-\frac{c_{1}^{2}}{F(\phi)}}} d \phi\right)\right]$

3.4 The $a(t)=e^{\lambda t^{\mu}}$ case

In this section we examine the effect of a scale factor of the form $a(t)=e^{\lambda t^{\mu}}$ with $\mu \neq 1$ (remember that the $\mu=1$ case was studied separately in Sect. 3.2). For $\lambda<0$ the scale factor can be said that follows a generalized normal distribution (the particular constant factor needed to multiply the exponent can be provided by scaling appropriately the spatial distance $r$ in the metric). With this specific example we want to demonstrate that the method we propose can lead to analytic solution even under the assumption of more complicated scale factors. In what regards the singularities of the space-time, it can be observed from the scalar curvature

$R=6 \lambda \mu t^{\mu-2}\left(\mu-1+2 \lambda \mu t^{\mu}\right)$,

$t=\sqrt[\mu]{-\frac{\mu-1}{3 \lambda \mu}+\frac{V(\phi)}{3 \lambda^{2} \mu^{2}} \sqrt[\mu /(2-\mu)]{-\frac{\mu-1}{3 \lambda \mu}+\frac{V(\phi)}{3 \lambda^{2} \mu^{2}} \sqrt[\mu /(2-\mu)]{-\frac{\mu-1}{3 \lambda \mu}+\frac{V(\phi)}{3 \lambda^{2} \mu^{2}} \ldots}}}$

that the latter diverges at $t \rightarrow \infty$ when $\mu>1$ and at $t=0$ when $\mu \in(-\infty, 2)-\{1\}$. With this choice of a scale factor equation (3.2) is written as an arbitrary power $\mu$. The solution can be given in terms of nested radicals in a manner similar that the solution of the more general equation of the form [60]

$\alpha t^{2 \mu}+\beta t^{\mu}+\gamma=t^{\nu}$.

In our case we have $\nu=2$ and $\gamma=0$. This value for $\gamma$ leads us to a small modification of the process of deriving the solution in comparison to what is presented in [60]. We first notice that $(3.45)$ can be written as

$$
\begin{aligned}
t^{\mu} & =-\frac{\mu-1}{3 \lambda \mu}+\frac{V(\phi)}{3 \lambda^{2} \mu^{2}} t^{2-\mu} \Rightarrow \\
t & =\sqrt[\mu]{-\frac{\mu-1}{3 \lambda \mu}+\frac{V(\phi)}{3 \lambda^{2} \mu^{2}} t^{2-\mu}} .
\end{aligned}
$$

We raise this last relation to the power $2-\mu$ (we remind that we assume $\mu \neq 2$ ) and we obtain

$t^{2-\mu}=\sqrt[\mu /(2-\mu)]{-\frac{\mu-1}{3 \lambda \mu}+\frac{V(\phi)}{3 \lambda^{2} \mu^{2}} t^{2-\mu}}$.

This means that the $t$ in (3.46) may now be given in terms of an infinite number of radicals through successive substitutions of (3.47). In particular we get:

$F(\phi)=-\frac{c_{1}^{2} e^{-6 \lambda t^{2}} V^{\prime}(\phi)^{2}}{\lambda^{2}(\mu-1)^{2} \mu^{2} t^{2(\mu-3)}\left(6 \lambda \mu t^{\mu}+\mu-2\right)^{2}+\left(2 V(\phi)-24 \lambda^{2} t^{2}\right) V^{\prime}(\phi)^{2}}$,

$\frac{3 \lambda^{2} \mu^{2}}{V(\phi)} t^{2 \mu}+\frac{\lambda(\mu-1) \mu}{V(\phi)} t^{\mu}=t^{2}$

We need to distinguish two cases depending on whether $\mu=$ 2 or not, something that is going to become obvious in the subsequent analysis.

\subsubsection{Case $\mu \neq 1,2$}

In this setting it is interesting to observe that the above equation can be solved algebraically with respect to $t$ even for
By obtaining the expression for $\dot{\phi}$ from the time derivative of (3.45) and by using Eq. (3.1) into the constraint (2.7a) we are led to

where $t$ is understood to be given in terms of $V(\phi)$ from (3.48). So $F(\phi)$ and $V(\phi)$ need to also be related with an expression involving nested radicals in order to acquire a solution of the form $a(t)=e^{\lambda t^{\mu}}$ with $\mu \neq 2$. We now proceed to complete this section with the study of the system when $\mu=2$.

\subsubsection{The $\mu=2$ case}

This situation corresponds to a normal distribution scale factor whenever $\lambda<0$. However, in what follows we make no particular assumption over the sign of $\lambda$. By setting $\mu=2$ 
Table 1 Relation between functions $F(\phi)$ and $V(\phi)$ for specific functional forms of the scale factor $a(t)$

\begin{tabular}{lll}
\hline$a(t)$ & $F-V$ relation & $F$ arbitrary \\
\hline$t^{\sigma}$ & Equation (3.7) if $\sigma \neq \frac{1}{3}$ & $V(\phi)=0$ if $\sigma=\frac{1}{3}$ \\
$\cosh ^{\alpha}(t)$ & Equation (3.30) if $\alpha \neq \frac{1}{3}$ & $V(\phi)=\frac{1}{3}$ if $\alpha=\frac{1}{3}$ \\
$\sinh ^{\alpha}(t)$ & Equation (3.39) if $\alpha \neq \frac{1}{3}$ & $V(\phi)=\frac{1}{3}$ if $\alpha=\frac{1}{3}$ \\
$e^{\lambda t^{\mu}}$ & $(3.49)$ if $\mu \neq 1,2$ or (3.52) if $\mu=2$ & $V(\phi)=3 \lambda^{2}$ if $\mu=1$ \\
\hline
\end{tabular}

in (3.45) we immediately see that it results in

$t= \pm \frac{\sqrt{V(\phi)-2 \lambda}}{2 \sqrt{3} \lambda}$

At the same time from its time derivative we get

$\dot{\phi}=\frac{24 \lambda^{2} t}{V^{\prime}(\phi)}$

By following the exact same procedure as in the previous cases, the above relations help us arrive at

$F(\phi)=\frac{c_{1}^{2} e^{1-\frac{V(\phi)}{2 \lambda}} V^{\prime 2}(\phi)}{96 \lambda^{3}-4 \lambda V^{\prime 2}-48 \lambda^{2} V(\phi)}$.

We study a quick example that demonstrates the application of relation (3.52).

a. Example: power Law potential.

We choose to set $V(\phi)=V_{0} \phi^{\kappa}+\Lambda$, where $\Lambda$ is a constant. According to (3.52) the compatible $F(\phi)$ function, for the scale factor we want, is

$F(\phi)=\frac{c_{1}^{2} \kappa^{2} V_{0}^{2} e^{1-\frac{\Lambda+V_{0} \phi^{\kappa}}{2 \lambda}} \phi^{2(\kappa-1)}}{96 \lambda^{3}-4 \kappa^{2} \lambda V_{0}^{2} \phi^{2(\kappa-1)}-48 \lambda^{2}\left(\Lambda+V_{0} \phi^{\kappa}\right)}$.

As in the process of deriving the solutions in the previous examples, we need to use (3.1) and (3.53) into the constraint equation and solve algebraically with respect to $\dot{\phi}$. Substitution of this result into Eq. (2.7b) for the given form of the potential $V(\phi)$ brings about the solution

$\phi(t)=\left(\frac{2 \lambda-\Lambda+12 \lambda^{2} t^{2}}{V_{0}}\right)^{1 / \kappa}$.

The above result leads (3.1) to yield

$$
\begin{aligned}
\psi(t)= & \psi_{0}-\int \frac{4 \lambda e^{3 \lambda t^{2}}}{c_{1}}\left[\frac{144 \lambda^{3} t^{2} V_{0}^{-2 / \kappa}}{\kappa^{2}}\right. \\
& \left.\times\left(2 \lambda\left(6 \lambda t^{2}+1\right)-\Lambda\right)^{\frac{2}{\kappa}-2}+1\right] d t .
\end{aligned}
$$

Thus, we derived the solution that allows for $a(t)=e^{\lambda t^{2}}$ when a power law potential is present.

We summarise all the results obtained in this section, involving the effect that the various scale factors have in the relation between $F(\phi)$ and $V(\phi)$, in Table 1 .

\section{Stability of the scaling solutions for the exponential potential}

We now proceed by studying the stability of the scaling solutions in the case where $V(\phi)$ and $F(\phi)$ are exponential functions, i.e. $V(\phi)=V_{0} e^{\lambda \phi}$ and $F(\phi)=F_{0} e^{\mu \phi}$. In this consideration the scalar field theory is reduced to the Chiral cosmology.

We introduce the new dimensionless variables [61] (where for the lapse function we assume $N=1$ ).

$x=\frac{\dot{\phi}}{\sqrt{6} H}, \quad y=\frac{\sqrt{V(\phi)}}{\sqrt{3} H}, \quad z=\frac{\sqrt{F(\phi)} \dot{\psi}}{\sqrt{6} H}$,

where $H=\frac{\dot{a}}{a}$. The field equations are written as the following system

$x^{2}+y^{2}+z^{2}-1=0$

$\frac{d x}{d \tau}=\frac{3}{2} x\left(x^{2}+z^{2}-y^{2}-1\right)+\frac{\sqrt{6}}{2}\left(\mu z^{2}-\lambda y^{2}\right)$,

$\frac{d y}{d \tau}=\frac{3}{2} y\left(x^{2}+z^{2}-y^{2}+1\right)+\frac{\sqrt{6}}{2} x y \lambda$,

$\frac{d z}{d \tau}=\frac{3}{2} z\left(x^{2}+z^{2}-y^{2}-1\right)-\frac{\sqrt{6}}{2} x z \mu$.

in which the new independent variable $\tau$ is defined as $\tau=$ $\ln (a(t))$.

From (4.2) it is clear that the variables $\{x, y, z\}$ evolve on a three-dimensional sphere with $y \geq 0$. Moreover, every critical point of the latter system corresponds to a phase where the scale factor $a(t)$ is given by the expressions $a(t)=$ $t^{\frac{2}{3\left(1+w_{t o t}\right)}}$ when $w_{t o t} \neq-1$ or $a(t)=e^{H_{0} t}$ when $w_{t o t}=$ -1 in which $w_{\text {tot }}$ is the equation of state parameter for the effective fluid defined as

$w_{t o t}(x, y, z)=x^{2}+z^{2}-y^{2}$. 
The critical points of the system (4.2)-(4.5) are determined to be

$$
\begin{aligned}
& P_{A}^{( \pm)}=( \pm 1,0,0), \quad P_{B}=\left(-\frac{\lambda}{\sqrt{6}}, \frac{\sqrt{6-\lambda^{2}}}{6}, 0\right), \\
& P_{C}^{( \pm)}=\left(-\frac{\sqrt{6}}{\lambda+\mu}, \sqrt{\frac{\mu}{\lambda+\mu}}, \pm \frac{\sqrt{\lambda^{2}+\lambda \mu-6}}{\lambda+\mu}\right) .
\end{aligned}
$$

Points $P_{A}^{( \pm)}$and $P_{B}$ correspond to the scaling solution where the field $\psi$ does not contribute to the evolution of the universe. Points $P_{A}^{( \pm)}$and $P_{B}$ are defined in the surface $z=0$ and are given in [61]. Points $P_{C}^{( \pm)}$are the new points that emerge from our analysis. We continue with the discussion of the physical quantities of the critical points as also with their stability. In order to study the latter, we reduce the dynamical system into a two-dimensional system given by the equations (4.3) and (4.4), where from (4.2) it follows $z^{2}=1-x^{2}-y^{2}$.

As far as the points $P_{A}^{( \pm)}$are concerned, the universe is dominated by the kinetic term of the scalar field $\phi$, i.e. $w_{\text {tot }}=$ 1. The eigenvalues of the linearized system are determined to be

$e_{1}\left(P_{A}^{( \pm)}\right)=3, e_{2}\left(P_{A}^{( \pm)}\right)=3 \pm \frac{\sqrt{6}}{2} \lambda$

from where we infer that the points are always unstable because $e_{1}\left(P_{A}^{( \pm)}\right)$is always positive.

Point $P_{B}$ exists only when $\lambda^{2}<6$ and describes a scaling solution where $w_{\text {tot }}=-1+\frac{\lambda^{2}}{3}$. The eigenvalue of the linearized system are determined to be

$e_{1}\left(P_{B}\right)=-3+\lambda^{2}, e_{2}\left(P_{B}\right)=-3+\frac{\lambda^{2}}{2}$,

from where we conclude that the solution at point $P_{B}$ is stable when $\lambda^{2}<3$. The universe is accelerated when $\lambda^{2}<2$.

In what regards the new points $P_{C}$, attained from our analysis, they describe a scaling solution with $w_{\text {tot }}=$ $-1+\frac{2 \lambda}{\lambda+\mu}$ which corresponds to an accelerated universe when $\left\{\mu<0, \frac{\mu}{2}<\lambda<|\mu|\right\}$ or $\left\{\mu>0,-\mu<\lambda<\frac{\mu}{2}\right\}$. This is the solution we previously derived. It is important to mention that the points exist when $\lambda \neq-\mu$ and $\{\mu<0, \lambda \leq-\sqrt{6}\}$ and $\left\{-\sqrt{6}<\lambda<0, \mu<\frac{6-\lambda^{2}}{\lambda}\right\}$ from where we infer that $w_{\text {tot }}<-\frac{1}{3}$ when $\{\lambda \leq-\sqrt{3}, \mu<\lambda\}$ or $\left\{-\sqrt{3}<\lambda<0, \mu<\frac{6-\lambda^{2}}{\lambda}\right\}$. As far as the stability is concerned, because of the nonlinearity dependence of the eigenvalues $e_{1}\left(P_{C}\right), e_{2}\left(P_{C}\right)$ on the free parameters $\mu$, and $\lambda$, we solve numerically the conditions where $e_{1}\left(P_{C}\right)<$ $0, e_{2}\left(P_{C}\right)<0$, and derive when the points are attractors. The region in the space $\{\lambda, \mu\}$ where the points are attractors are given in Fig. 1.

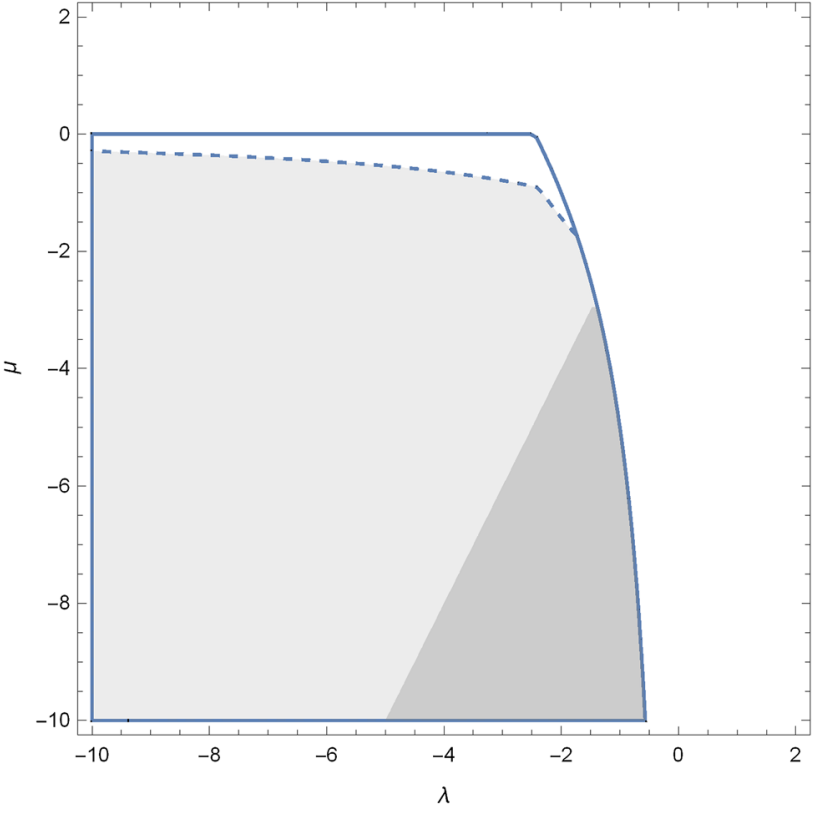

Fig. 1 Region plot in the space of variables $\{\lambda, \mu\}$ for the eigenvalues $e_{1}\left(P_{C}\right), e_{2}\left(P_{C}\right)$. With the solid line is the boundary where the points exist. Dashed line describe the boundary where the points are attractors. The ope gray area corresponds to the case where $w_{t o t}>-\frac{1}{3}$, while the dark gray area describe the values of the parameters $\{\lambda, \mu\}$ in which $w_{\text {tot }}<-\frac{1}{3}$

In Fig. 2 we present the phase space diagram in the variables $\{x, y, z\}$ for the dynamical system of our consideration for two set of the values $\lambda$ and $\mu$. More specifically, the plots are for the set $(\lambda, \mu)=(-1,2)$ where the only stable point is $P_{B}$ and for $(\lambda, \mu)=(-3,-4)$ where points $P_{C}^{( \pm)}$are the two attractors of the dynamical system.

We summarize the results of the critical point analysis in Table 2 .

Let us now discuss in some detail the new critical point $P_{C}$ : The cosmological fluid source at this critical point has an equation of state parameter $w_{t o t}=-1+\frac{2 \lambda}{\lambda+\mu}$, therefore we can easily calculate that

$a(t)=t^{\frac{1}{3}\left(1+\frac{\mu}{\lambda}\right)}, \quad H(t)=\frac{\lambda+\mu}{3 \lambda} \frac{1}{t}$.

Moreover, from (4.3)-(4.5) and (4.1) it follows that at the critical point

$\frac{d x}{d \tau}=0, \quad \frac{d y}{d \tau}=0, \quad \frac{d z}{d \tau}=0$

that is

$\dot{\phi}=x_{0} \sqrt{6} H, \quad V_{0} e^{\lambda \phi}=\left(y_{0}\right)^{2} H^{2}, \quad \dot{\psi}=\sqrt{6} z_{0} e^{-\frac{1}{2} \mu \phi} H$

which implies 

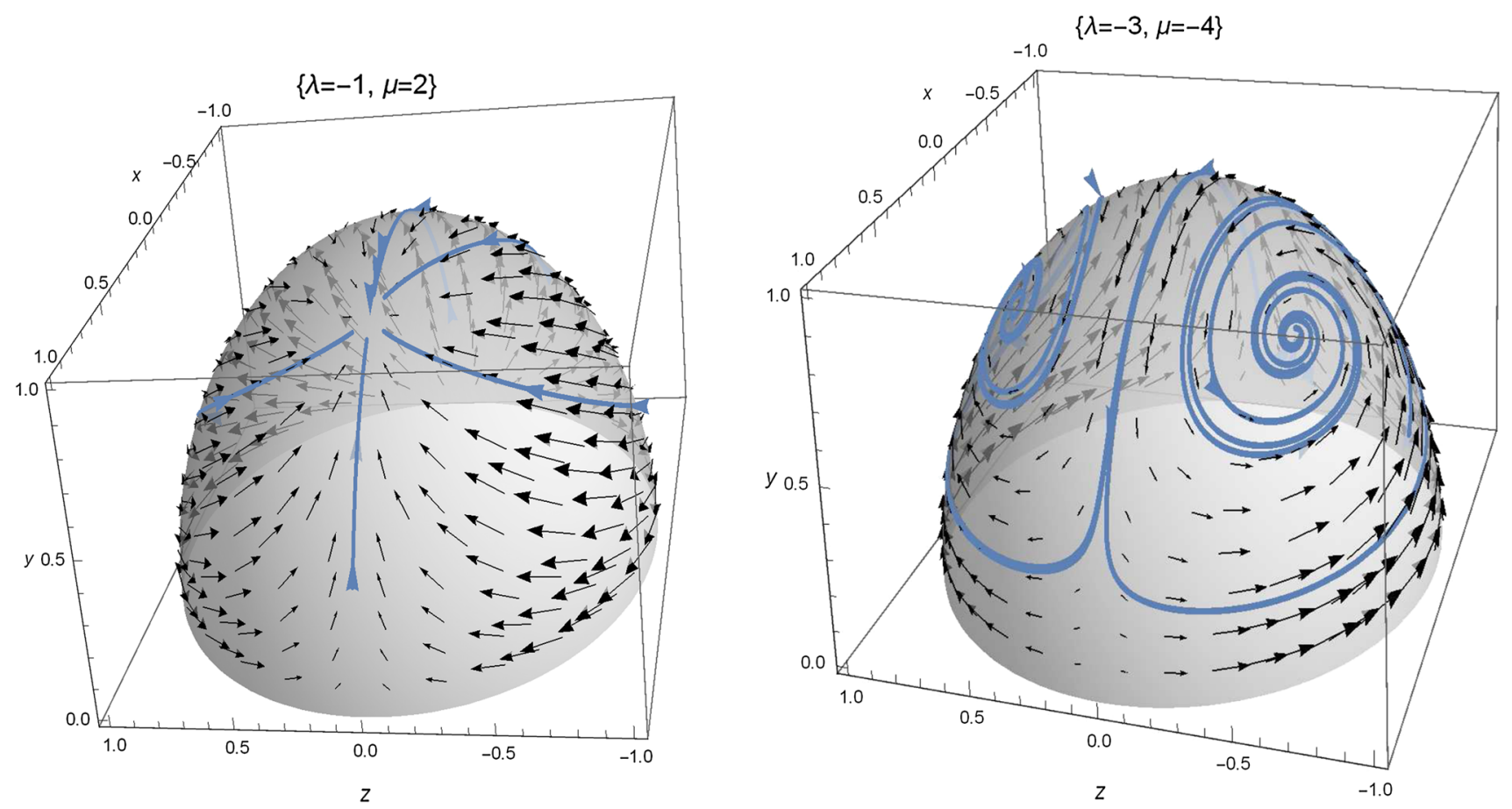

Fig. 2 Phase space diagram for the dynamical system (4.3)-(4.5) for two different set of the free parameters $\lambda$ and $\mu$. Left figure is for $(\lambda$, $\mu)=$ $(-1,2)$ where point $P_{B}$ is the unique attractor while right figure is for $(\lambda, \mu)=(-3,-4)$ and points $P_{C}^{ \pm}$are the attractors of the dynamical system

Table 2 Critical points of the dynamical system

\begin{tabular}{|c|c|c|c|c|}
\hline Point & $(x, y, z)$ & Existence & Stability & Acceleration \\
\hline$P_{A}^{( \pm)}$ & $( \pm 1,0,0)$ & Always & Unstable & No \\
\hline$P_{B}$ & $\left(-\frac{\lambda}{\sqrt{6}}, \frac{\sqrt{6-\lambda^{2}}}{6}, 0\right)$ & $\lambda^{2}<6$ & Stable: $\lambda^{2}<3$ & $\lambda^{2}<2$ \\
\hline$P_{C}^{( \pm)}$ & $\left(-\frac{\sqrt{6}}{\lambda+\mu}, \sqrt{\frac{\mu}{\lambda+\mu}}, \pm \frac{\sqrt{\lambda^{2}+\lambda \mu-6}}{\lambda+\mu}\right)$ & $\lambda \neq-\mu\{\mu<0, \lambda \leq-\sqrt{6}\}\left\{-\sqrt{6}<\lambda<0, \mu<\frac{6-\lambda^{2}}{\lambda}\right\}$ & See Fig. 1 & See Fig. 2 \\
\hline
\end{tabular}

$$
\begin{aligned}
\dot{\phi} & =\sqrt{6} x_{0} \frac{\lambda+\mu}{3 \lambda} \frac{1}{t}, \quad e^{\lambda \phi}=\left(y_{0} \frac{\lambda+\mu}{3 \lambda}\right)^{2} \frac{1}{t^{2}}, \dot{\psi} \\
& =\sqrt{6} z_{0} \frac{\lambda+\mu}{3 \lambda} t^{-\frac{\sqrt{6} x_{0}}{6} \frac{\mu}{\lambda}(\lambda+\mu)-1}
\end{aligned}
$$

we finally find

$$
\begin{aligned}
\phi(t)-\phi_{0}= & \sqrt{\frac{2}{3}} \frac{x_{0}(\lambda+\mu)}{\lambda} \ln t, \psi(t) \\
& -\psi_{0}=\sqrt{\frac{2}{3} \frac{\lambda+\mu}{\mu}} z_{0} e^{-\frac{\mu}{2} \phi_{0}} t^{\frac{\mu}{\lambda}}
\end{aligned}
$$

with the constants $x_{0}$ and $y_{0}$ being constrained as $x_{0}=$ $-\frac{\sqrt{6}}{\lambda+\mu}, \quad\left(y_{0}\right)^{2}=\frac{9 \lambda^{2} V_{0}}{(\lambda+\mu)^{2}} e^{\lambda \phi_{0}}$. This is precisely the exact solution (3.15), (3.16) determined before.

As we discussed above, when $\frac{2 \lambda}{\lambda+\mu}<\frac{2}{3}$, the cosmological solution at point $P_{C}$ describes an accelerated universe, while the attractor where the two scalar fields survive has been used to describe the inflationary era. When the field space is a hyperbolic plane [62], the model is called hyperinflation. There are various differences between hyperinflation and the slow-roll inflation [63], for an elaborate discussion see [62]. Remark that point $P_{B}$ describe the slow-roll inflation era since only field $\phi$ contribute to the cosmological evolution. On the other hand, $P_{C}$ corresponds to the two field case and particularly to a solution that emerges for a coupling function $F(\phi)=$ $F_{0} e^{\mu \phi}$, which makes the two dimensional field space hyperbolic.

Recently in [64] the dynamics of the later cosmological model has been studied by using a different set of variables. Our analysis can be directly compared with that of [64] and shows that the use of a different set of variables leaves the dynamics invariant. The critical point $P_{C}$ corresponds to the point $(x, y)_{\text {hyper }}$ of [64] (corresponding to the $x, z$ of our analysis) where the spiralling behaviour, as described in [64], is given by eigenvalues with nonzero imaginary part; such a behaviour is presented in Fig. 2. 


\section{Conclusion}

We considered a two-scalar field cosmological model with a mixed kinetic term. More specifically, we assume that the matter source of the Einstein's General Relativity is described by two scalar field which they have an interacting term in the kinetic part of the scalar field Lagrangian. For this model, we investigate the existence of some exact solutions for the scale factor which describe different phases in the evolution of the universe.

In particular, we proved the existence of power-law solutions, de Sitter universe, asymptotic de Sitter universe with or without initial singularity and for a scale factor that depends on the exponential function of arbitrary powers of the time variable. For each specific solution we found the constraining condition between the two unknown functions of the theory so that it would be admissible. These unknown functions are the scalar field potential $V(\phi)$ and the one that provides the interaction in kinetic term $F(\phi)$. A special case of the model which we considered is the Chiral cosmology, which can be seen as a unified dark energy and dark matter model. We also demonstrated the validity of our result by providing several examples in which we choose the potential function $V(\phi)$ and derive the compatible $F(\phi)$ for which the space-time of our choice is admissible.

For the Chiral cosmological model and for the exponential potential we apply the critical point analysis in order to determine the stability of the scaling solutions. We found that the dynamical system admits five critical points where the three of them correspond to the quintessence model of General Relativity. The two new critical points describe scaling solutions and by our analysis we were able to study the stability of the exact solutions.

Apart from the approach that we followed here in which, when certain conditions are set over the scale factor, the potential is related to the cosmological time through Eq. (3.2), a different procedure can also be applied: It is known that, in the case of a single scalar field, when the latter is utilized as an effective time variable, you can express the potential as a function of quantities containing only $\phi[63,65]$. This is made possible with the use of the equations of motion and is called the Hamilton-Jacobi method. A similar methodology has been put in use recently in $[66,67]$ for the case of multiple scalar fields where the potential is expressed in terms of the fields and their first derivatives. Especially in [67] - and for a two scalar field system - the adoption of the a shiftsymmetric orbital inflation condition leads to expressing the potential as a pure function of the two fields while at the same time providing with an exact solution. It is interesting to note that also in this approach one obtains a relation between the potential and the kinetic coupling function, although under a different context since the potential is assumed to depend on both fields.
In a future work we plan to investigate the case where the two scalar fields are interacting also in the potential term, as also when they are nonminimally coupled with the gravity [68].

Data Availability Statement This manuscript has no associated data or the data will not be deposited. [Authors' comment: This is a work in theoretical cosmology and thus there is no data to submit.]

Open Access This article is distributed under the terms of the Creative Commons Attribution 4.0 International License (http://creativecomm ons.org/licenses/by/4.0/), which permits unrestricted use, distribution, and reproduction in any medium, provided you give appropriate credit to the original author(s) and the source, provide a link to the Creative Commons license, and indicate if changes were made.

Funded by $\mathrm{SCOAP}^{3}$.

\section{References}

1. D.J. Eisenstein et al., SDSS Collaboration. Astrophys. J. 633, 560 (2005)

2. C.L. Bennett et al., WMAP Collaboration. Astrophys. J. Suppl. 208, 20 (2013)

3. P.A.R. Ade et al., Planck Collaboration. Astron. Astrophys. A $\mathbf{5 9 4}$ $13(2016)$

4. S. Weinberg, Rev. Mod. Phys. 61, 1 (1989)

5. P.J. Peebles, B. Ratra, Rev. Mod. Phys. 75, 559 (2003)

6. B. Ratra, P.J.E. Peebles, Phys. Rev D. 37, 3406 (1988)

7. N. Dimakis, A. Karagiorgos, A. Zampeli, A. Paliathanasis, T. Christodoulakis, Petros A. Terzis, Phys. Rev. D 93, 123518 (2016)

8. C.H. Brans, R.H. Dicke, Phys. Rev. 124, 925 (1961)

9. J. O'Hanlon, Phys. Rev. Lett. 29, 137 (1972)

10. G.W. Hordenski, Int. J. Theor. Phys. 10, 363 (1974)

11. C. Deffayet, D.A. Steer, Class. Quant. Gravit. 30, 214006 (2013)

12. J.D. Barrow, Phys. Rev. D 85, 047503 (2012)

13. T.P. Sotiriou, Modifications of Einstein's Theory of Gravity at Large Distances, in Lecture Notes in Physics, vol. 892, ed. by E. Papantonopoulos (Springer, New York, 2015)

14. A.D. Linde, Phys. Lett. B 129, 177 (1983)

15. J.D. Barrow, Phys. Rev. D 48, 1585 (1993)

16. J.D. Barrow, P. Saich, Class. Quant. Gravit. 10, 279 (1993)

17. P.J. Peebles, B. Ratra, Astrophys. J. Lett. 325, L17 (1988)

18. S. Tsuwikawa, Class. Quant. Gravit. 30, 214003 (2013)

19. V. Sahni, L.M. Wang, Phys. Rev. D 62, 103517 (2000)

20. L.A. Ureña-López, J. Phys. Conf. Ser. 761, 012076 (2016)

21. D. Bertacca, N. Bartolo, A. Diaferio, S. Matarrese, JCAP 08, 023 (2008)

22. D. Bertacca, S. Matarrese, M. Pietroni, Mod. Phys. Lett. A 22, 2893 (2007)

23. H. Kim, Mon. Not. Roy. Astron. Soc. 364, 813 (2005)

24. I. Leanizbarrutia, A. Rozas-Fernadez, I. Tereno, Phys. Rev. D 96, 023503 (2017)

25. A.D. Lindle, Phys. Rev. D 49, 784 (1994)

26. E.J. Copeland, A.R. Liddle, D.H. Lyth, E.W. Steward, D. Wands, Phys. Rev. D 49, 6410 (1994)

27. S.A. Kim, A.R. Liddle, Phys. Rev. D 74, 023513 (2006)

28. D. Wands, Lect. Notes Phys. 738, 275 (2008)

29. P. Carrilho, D. Mulryne, J. Ronaye, T. Tenkanen, JCAP 06, 032 (2018)

30. P. Christodoulidis, D. Roest, E.I. Sfakianakis, Attractos, bifucarions and curvature in multi-filed inflation. arXiv:1903.03513

31. K. Maeda, S. Mizuno, R. Tozuka, Phys. Rev. D 98, 123530 (2018)

32. T. Kobayashi, O. Seto, T.H. Tatsuishi, PTEP 12, 123 B04 (2017) 
33. S.V. Chervon, Quant. Mater. 2, 71 (2013)

34. S. Tsujikawa, Phys. Rev. D 73, 103504 (2006)

35. Y. Li, Int. J. Mod. Phys. D 26, 1750164 (2017)

36. A.A. Andrianov, O.O. Novikov, C. Lan, Theor. Math. Phys. 184, 1224 (2015)

37. Y.F. Cai, E.N. Saridakis, M.R. Setare, J.-Q. Xia, Phys. Rep. 493, 1 (2010)

38. E.N. Saridakis, J.W. Weller, Phys. Rev. D 81, 123523 (2010)

39. S.V. Chervon, Russ. Phys. J. 38, 539 (1995)

40. S.V. Chervon, S.D. Maharaj, A. Beesman, A.S. Kubasov, Gravit. Cosmol. 20, 176 (2014)

41. S.V. Chervon, Russ. Phys. J. 39, 139 (1996)

42. S.V. Ketov, Quantum Non-linear Sigma Models (Springer, Berlin, 2000)

43. J. Lee, T.H. Lee, T. Moon, P. Oh, Phys. Rev. D 80, 0656016 (2009)

44. Y. Zhang, Y.-G. Gong, Z.-H. Zhu, Phys. Lett. B 688, 13 (2010)

45. T. Chiba, A. De Felice, S. Tsujikawa, Phys. Rev. D 90, 023516 (2014)

46. A.S. Sakharov, MYu. Khlopov, Phys. Atom. Nucl. 56, 412 (1993)

47. P.H.R.S. Moraes, J.R.L. Santos, Phys. Rev. D. 89, 083516 (2014)

48. D. Bazeia, L. Losano, J.R.L. Santos, Phys. Lett. A 377, 1615 (2013)

49. I.Ya. Aref'eva, N.V. Bulatov, SYu. Vernov, Theor. Math. Phys. 163, 788 (2010)

50. A. Paliathanasis, M. Tsamparlis, Phys. Rev. D 90, 43529 (2014)

51. J. Socorro, O.E. Nunez, R. Hernandez-Jimenez, Adv. Math. Phy. 3468381 (2018)

52. S.D. Maharaj, A. Beesham, S.V. Chernov, A.S. Kubasov, Gravit. Cosmol. 23, 375 (2017)
53. A. Paliathanasis, G. Leon, S. Pan, Exact solutions in multi-scalar field cosmology. arXiv:1811.1091

54. A. Paliathanasis, S. Pan, S. Pramanik, Class. Quant. Gravit. 32, $245006(2015)$

55. A.A. Coley, R.J. van den Hoogen, Phys. Rev. D 62, 023517 (2000)

56. F.-W. Chen, B.-M. Gu, Y.-X. Liu, EPJC 78, 131 (2018)

57. H. Mohseni Sadjadi, M. Honardoost, H.R. Sepangi, Phys. Dark Univ. 14, 40 (2016)

58. B. Feng, X. Wang, X. Zhang, Phys. Lett. B 607, 35 (2005)

59. Z.-K. Guo, Y.-S. Piao, X. Zhang, Y.-Z. Zhang, Phys. Lett. B 608, 177 (2005)

60. N. Bagis, Solution of polynomial equations with nested radicals. arXiv:1406.1948 [math-GM]

61. E.J. Copeland, A. Liddle, D. Wands, Phys. Rev. D 57, 4686 (1998)

62. A.R. Brown, Phys. Rev. Lett. 121, 251601 (2018)

63. A. Linde, Phys. Lett. B 108, 389 (1982)

64. P. Christodoulidis, D. Roest, E.I. Sfakianakis, Scaling attractors in multi-field inflation. arXiv: 1903.06116

65. D.S. Salopek, J.R. Bond, Phys. Rev. D 42, 3936 (1990)

66. A. Achucarro, S. Cespedes, A.C. Davis, G.A. Palma, Phys. Rev. Lett. 122, 191301 (2019)

67. A. Achúcarro, E.J. Copeland, O. Iarygina, G.A. Palma, D.G. Wang, Y. Welling, Shift-symmetric orbital inflation: single field or multifield? arXiv: 1901.03657

68. D.I. Kaiser, Phys. Rev. D 81, 084044 (2010) 